\title{
Cortical plasticity in an early blind musician: an fMRl study
}

\author{
David A. Ross ${ }^{\mathrm{a}, *}$, Ingrid R. Olson ${ }^{\mathrm{b}}$, John C. Gore \\ ${ }^{a}$ Department of Diagnostic Radiology, Yale School of Medicine, New Haven, CT, USA \\ ${ }^{\mathrm{b}}$ Department of Psychology, University of Pennsylvania, Philadelphia, PA, USA \\ ${ }^{\mathrm{c}}$ Department of Radiology and Radiological Sciences, Vanderbilt University School of Medicine, Nashville, TN, USA
}

Received 10 October 2002; accepted 14 January 2003

\begin{abstract}
Many studies have examined tactile perception and simple auditory perception in the blind, but none have previously investigated the neural basis of musical ability in this group. This topic is of particular interest because it has been suggested that early blind individuals may possess advanced musical skills (such as absolute pitch). Presumably, these skills could be the result of neural plasticity. It has been hard to empirically assess this claim because of the difficulty in recruiting an adequate number of subjects for use in a conventional paradigm. In the present paper, we report data from a congenitally blind musician, subject ML. Behavioral tests show that she possessed absolute pitch abilities that were similar to those of a reference group of AP musicians with normal vision. To examine the neural basis of her abilities we then tested subject ML and five control subjects using an fMRI paradigm. A regions-of-interest analysis found that similar areas (the right secondary auditory cortex, left IFG, left SMG) were activated in ML and the control subjects, to a similar degree, in response to music processing. However, ML showed additional activations in left parietal association cortices and extrastriate regions of the occipital lobe. Subject ML's data are consistent with a vast body of literature on blindness-induced plasticity. They extend these findings by demonstrating that cortical plasticity may underlie special musical skills as well. These data illustrate the potential value of case studies to investigate particularly rare phenotypes. (C) 2003 Elsevier Inc. All rights reserved.
\end{abstract}

Keywords: Blind; Plasticity; Music; Absolute pitch (AP)

\section{Introduction}

Music has often been postulated as an area of advanced competence in blind individuals, perhaps due to high profile individuals such as Ray Charles and Stevie Wonder. It is plausible that this musical expertise arises from a learninginduced alteration in neural structures that is enhanced in part by the deprivation of sight and the subsequent reliance on audition. A substantial body of literature has been devoted to understanding this type of plasticity and the complex interplay that occurs between the negative impact of sensory deprivation and the simultaneous, compensatory, expansion of other brain regions.

Animal studies of visual deprivation have shown that changes occur both subcortically (e.g., in the superior colliculus $[24,37]$ and lateral geniculate nucleus $[20,23]$ ) as well as in the primary and secondary visual cortices $[5,22]$.

\footnotetext{
* Corresponding author. Tel.: +1-203-785-5296; fax: +1-203-7856534.

E-mail address: david.a.ross@yale.edu (D.A. Ross).
}

Further, functional studies of brain activity in early blind humans show that regions normally involved in visual association (including accessory visual cortex and parietal association areas) may be recruited to process both tactile $[6,9,29-31,36]$ and auditory $[1,16,17]$ stimuli. Plasticity has also been observed throughout the auditory pathway of both visually deprived animals $[12,14,25,28]$ and early blind humans $[6,17,26,36]$. While these studies elucidate some heightened auditory skills in blind individuals (e.g., enhanced spatial localization), relatively few paradigms have examined auditory processing as compared to somatosensory or visual systems. Moreover, to the best of our knowledge, no studies have addressed the functional plasticity associated with musical expertise in the blind.

Previously [27], we used functional magnetic resonance imaging (fMRI) to study the neural networks involved in intervalic pitch perception (i.e., the determination of frequency relationships) in musicians who were possessors or non-possessors of absolute pitch (AP or NAP). We hypothesized that differences in the auditory cortex may account for the basic, perceptual differences between AP and NAP 
musicians. Indeed, AP subjects demonstrated a highly consistent pattern of activation that was distinct from that of NAP subjects in the right auditory cortex. Additional activations were found in the left supramarginal gyrus (SMG) and the left inferior frontal gyrus (IFG).

The consistency of the pattern of activations in AP subjects led us to ask the question: would similar areas be recruited by early blind musicians who also possessed absolute pitch? If blind individuals with AP did not share the same auditory activation, it would suggest that a rearrangement of functional regions had caused them to develop this distinct phenotype. Alternatively, if early blind individuals shared similar basic activations with other AP musicians but also showed new activations not present in our previous study, it would suggest that these individuals may recruit additional cortical areas for musical processing when there is deprivation of a major sensory modality.

Unfortunately, it would be difficult to address this question in a conventional manner. Historically, the majority of studies that have investigated functional plasticity have used PET imaging, which requires a relatively large number of subjects per group. To recruit an adequate number of subjects with a specialized skill from within what is already a very small subset of the population would be prohibitve (consider that the prevalence of AP is estimated to be $\sim 1 / 10,000$ [2] and the prevalence of early blindness in the United States is probably less than 1/1,000 (see www. cdc.gov/ncbddd/); thus, if they were independent phenomena the overall prevalence would be $\sim 1 / 10,000,000$; given the most liberal estimate of dependence (cf. [38]), it would still be less than 1/5,000). Fortunately, the development of fMRI techniques has now made it possible to investigate functional activation in single subjects. While it is difficult to make broad conclusions based on a single individual, such data may still yield important insights into otherwise inaccessible phenomena.

In this paper, we used fMRI to investigate the neural basis of exceptional musical skills in a congenitally blind musician, subject ML. Our goal was to determine whether she used the same neural substrates during the performance of a specific, higher-order, musical task as subjects of similar ability but with normal vision. We used the same paradigm that we had tested previously with AP and NAP musicians. Briefly, this paradigm involved listening to five note sequences and naming them according to the rules of "moveable do" solfeggio. There was no visual stimulation and the control subjects had been asked to keep their eyes closed during all imaging conditions. The solfeggio task was chosen because it explicitly isolates the process of identifying the relationship between frequency stimuli without regard for the absolute frequency of the tones. It is important to emphasize that both AP possessors and nonpossessors must be skilled at such comparisons if they are to be successful musicians. This is true because music is fundamentally based on the relationships between notes rather than on the specific frequency values, per se. For example, different orchestras may tune to different frequency values of "A." Similarly, the correct frequency of the note "F\#" will differ for non-keyboard instruments depending on the key of the piece. Another example of the essential nature of relative tuning is in choral music: because choirs typically go flat as a piece progresses, it is imperative that musicians sing notes that are correctly tuned to their peers rather than to any absolute values. Thus, the ability to recognize the frequency of tones may be seen as an additional skill found in AP possessors- however, both AP and NAP musicians must develop a refined sense of relative pitch as well (cf. [3]). "Moveable Do" solfeggio is a natural extension of this skill with which all of our subjects were familiar.

In the current study, we were specifically interested in the three significant areas of activation found previously (the right secondary auditory cortex, left IFG, left SMG) and other cortical regions that are known, based on previous research, to be recruited specially by blind individuals performing sensory tasks (i.e., polymodal areas in the parietal lobe, and extrastriate cortex in the occipital lobe).

\section{Materials and methods}

\subsection{Subjects}

The subject, ML, was an eighteen year old, right handed, congenitally anophthalmic/microphthalmic woman. Since birth, she has had minimal residual light sensation in her right eye and no vision in her left eye. She began musical training in performance at age 3 and had nine years of experience on the trumpet (her primary instrument) and an additional 8 years of experience on the piano. She began studying music theory at age 6 and had a total of 10 years experience in the field. ML reported possessing AP and could not recall a time in life when she did not. She was aware of the experimental paradigm and signed a voluntary consent form for her participation.

Data from AP subjects with normal vision in the original experiment [27] were used for reference. The five subjects (4 male, $5 \mathrm{RH}$ ) had an average age of $37 \pm 11$ years (range 19-49) with an average of $26 \pm 10$ years of experience on their primary instrument and $17 \pm 15$ years of experience in music theory.

\subsection{Design and procedure}

\subsubsection{AP test}

In order to determine whether or not ML had true AP, she was administered a standard note naming test. The test required subjects to name 30 sinusoid tones and 30 piano tones (each of which were presented in three blocks of ten) that were played in rapid succession (note duration $=500$ $\mathrm{ms}$; inter-stimulus interval $=2000 \mathrm{~ms}$ ). Sinusoid tones were generated using SoundEdit II for the Macintosh. Piano tones were generated using the "Piano 1" setting of a Roland 


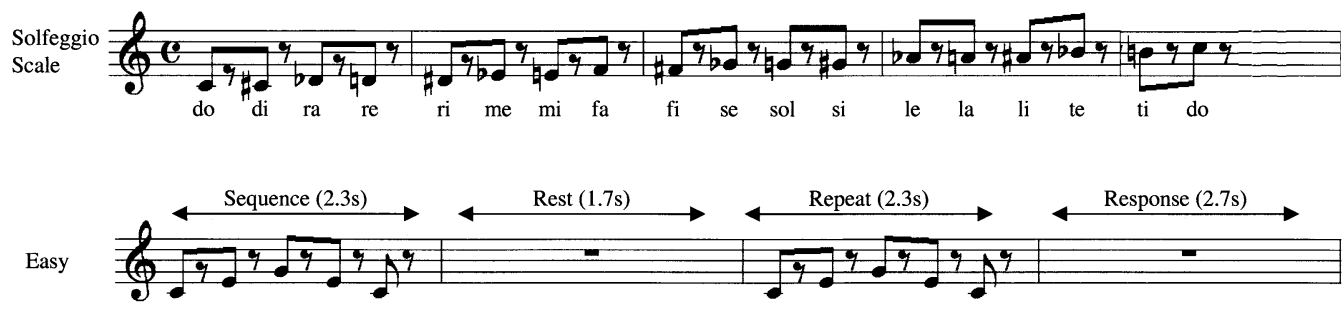

'do mi sol mi do'

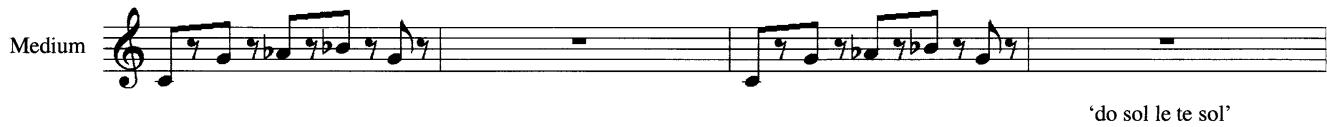

Hard

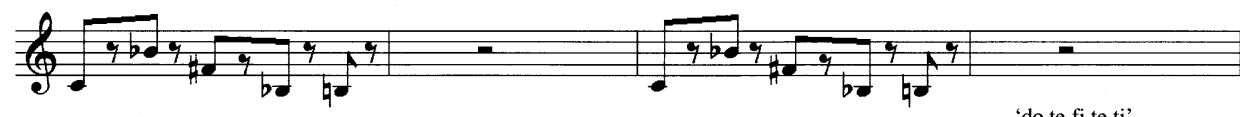

Fig. 1. Using "moveable-do" solfeggio, subjects were asked to label the first tone of each sequence as "do," and label the subsequent four notes according to their relationship to the tonic. A sample scale and representative sequences are shown in the key of "C," though sequences were evenly distributed in all 12 keys. Easy sequences consisted primarily of notes from the major, diatonic, scale. Medium sequences were drawn from the natural minor scale with relatively little mixture. Hard sequences were modeled after the rules of post-tonal theory and consisted of large, dissonant, intervals, with a high degree of chromaticism.

JV-50 synthesizer. Notes ranged from C2-C7 (65-2092 Hz) and were pseudo-randomly selected such that no note values repeated within a 5 note span and no successive notes were within 9 whole steps of each other. Stimuli were presented via Psyscope software [8] with an identical script for all subjects. Subjects were asked to respond with the note name (e.g., "C" or "F\#") but were not required to identify the note octave. Subjects were qualified as possessing AP if their overall accuracy exceeded $80 \%$.

\subsection{2. fMRI task}

For the experimental condition subjects were required to perform an analytical task that is familiar to most musicians- "moveable do" solfeggio (see Fig. 1). This task is designed to isolate the process of intervalic pitch perception- i.e., the ability to recognize the relationship between different frequencies without regard for their absolute values. For the reasons described above this skill is essential to musical processing and it is known to be possessed by both AP and NAP subjects. Each stimulus consisted of a five note musical sequence. For each sequence subjects were required to designate the first note as "do"- irrespective of its absolute frequency- and to label the subsequent four notes based on their scalar relationship to the tonic. Stimuli were divided into three levels of difficulty according to basic principles of music theory. Easy sequences consisted primarily of notes drawn from the major, diatonic, scale. Medium sequences were taken from the natural minor scale with relatively little mixture. Hard sequences were modeled after the rules of post-tonal theory and consisted of large, dissonant intervals with a high degree of chromaticism. Sequences were distributed equally across all keys such that the note value of "do," the first note in each sequence, varied at random. The stimuli were piano tones, as described in section B above.

The control task was an auditory test that was similar to the experimental task in every regard except that subjects were required to compare stimuli to each other without the use of any tonal information. To do this, five "pink" noise bursts were created, all of which had the same central frequency but differed in their spectral power distribution. A labeling system was created that was similar to the solfeggio scale ("bah," "dah," "fo," "kah," and "nay"), and the first note of every sequence was always "bah" (as the first note of every tonal sequence was "do"). Additionally, each sequence was shifted up or down by up to one whole step in pitch in order to simulate the experimental effect of different keys. Therefore, as in the experimental condition, rather than having subjects identify fixed entities, the emphasis of the task remained on determining their relative relationships. Subjects were trained on performance of the control task for approximately two hours in advance of the experiment, until they were both comfortable and proficient in their performance of the task. It was our goal to match performance on the control task to that of the medium solfeggio condition.

In direct comparison, the experimental and control conditions were matched in terms of primary auditory processing, short term memory, decision making, and naming of stimuli, thereby isolating the processes involved in assessing pitch relationships. Thus, subtraction of subject activation during the performance of the control task from subject activation during the performance of the musical tasks should reveal those regions of the brain that are involved in understanding the relationship between pitches. 
The timing of sequences was identical for both musical and control tasks. The note duration was $300 \mathrm{~ms}$ and the interval between notes was $200 \mathrm{~ms}$. Each sequence was repeated once (i.s.i. $=1700 \mathrm{~ms}$ ) and, following the second playing, subjects were given a brief period to respond $(\mathrm{t}=$ $2300 \mathrm{~ms}$ ). Stimuli were presented in pseudo-random block order ( 1 block $=2$ sequences) such that the different tasks (E, M, H, C) appeared with equal frequency in each position. Subjects were given one practice run, in magnet, in order to accustom themselves to the unfamiliar environment and additional background noise. Stimuli were played from Psyscope using a script that was identical for all subjects and were presented to the subjects through tight fitting headphones that were designed to minimize the sounds inherent to EPI imaging. Also, to the extent that background noise was perceived by the subjects, it was present to an equal extent in both musical and control tasks.

Eight scanning sequences (total length $=229 \mathrm{~s}$; task length $=222 \mathrm{~s}$ ) were conducted during which fMRI data were collected. In order to reduce motion artifact, subjects were asked to covertly state all responses. Following completion of the last scan, an additional run was conducted verbally, outside of the magnet, in order to determine performance accuracy.

\subsection{3. fMRI specifications}

Functional MRI scans were obtained and data were analyzed using image processing and statistical methods specifically adopted for fMRI. Gradient echo, echo planar imaging was used (repetition time (TR) $=1725 \mathrm{~ms}$; echo time $(\mathrm{TE})=45 \mathrm{~ms}$; flip angle $=60^{\circ}$ ) on a 1.5 Tesla GE magnet. Eight slices were acquired (thickness $=8 \mathrm{~mm}$; interslice interval $=1 \mathrm{~mm}$; 128 images per slice) in an axial orientation, parallel to the AC-PC plane, with the sixth slice aligned to the top of the corpus callosum (in plane resolution $1.56 \times 3.12 \mathrm{~mm}$ ). Functional images were co-aligned with a high-resolution anatomic scan taken in the same session (two dimensional spin echo sequence; TR $=500$ $\mathrm{ms}$; $\mathrm{TE}=13 \mathrm{~ms}$; number of excitations $=2$; matrix $256 \times$ 192 voxels).

\subsubsection{Data analysis}

Data were motion corrected using SPM software (Wellcome Department of Cognitive Neurology, London, UK). Activation from each musical condition was compared to the noise condition to generate parametric maps of activation for each subject; additionally, a comparison was made between the average musical activation and the noise condition. A detailed description of the statistical software package may be found in Skudlarski et al. [32].

For control subjects, activation maps and structural images were transformed into Talairach Space [34] and an average composite image was generated. Fig. 2 provides a scout view illustrating the exact location of Talairach slices.

A region of interest analysis (ROI) was also performed. ROIs were defined in standard Talairach space and were

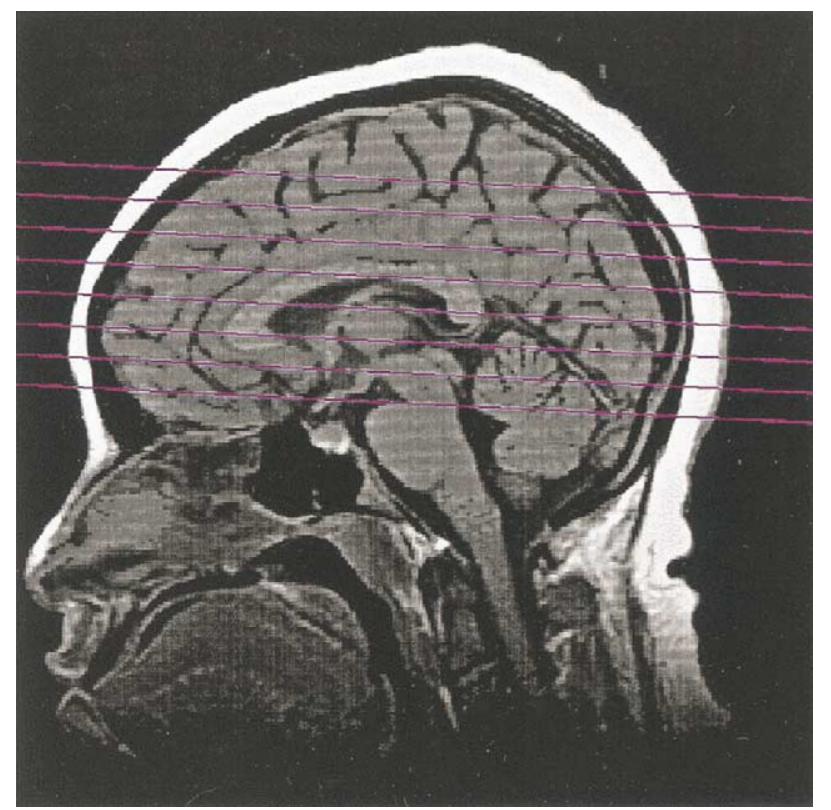

Fig. 2. Midline sagittal view illustrating the location of eight Talairach slices. Subsequent composite figures show slices in descending order, from upper left to lower right, where slices correspond to Talairach z-values of $50.2,41.1,31.9,22.9,13.7,4.6,-5.4,-16.1$.

determined by a radiologist before the experiment was conducted. The secondary auditory cortex ROI was the anterior superior and middle inferior aspect of the superior temporal gyrus. The ROI was 15 voxels wide, and an average of 12 voxels deep extending inferior through 2 slices. The IFG ROI was 20 voxels wide, and an average of 8 voxels deep, extending inferior through 3 slices. The SMG ROI was 20 voxels wide, and an average of 15 voxels deep, extending inferior through 4 slices. The angular gyrus ROI was 20 voxels wide. The ROI was an average of 10 voxels deep, extending inferior through 3 slices. The extrastriate ROI consisted of BA 18 and 19, was 35 voxels wide, an average of 15 voxels deep, and extended through 6 slices. The summed signal intensity of all activated voxels was calculated for each ROI and values were compared between subject ML and the five reference subjects using Sokal's transformed $t$ test for comparing a single specimen to a sample $^{1}[33]$.

${ }^{1}$ On pg. 230 of his text, Sokal explains the procedure for comparing a single data point to a sample. $t$-values are calculated using the following formula:

$$
t_{s}=\frac{Y_{1}-\bar{Y}_{2}-\left(\mu_{1}-\mu_{2}\right)}{s_{2} \sqrt{\frac{n_{2}+1}{n_{2}}}}
$$

where $\mathrm{Y}_{1}$ is the single specimen, $\bar{Y}_{2}$ is the sample mean, $\mathrm{s}_{2}$ is the sample standard deviation, $\mathrm{n}_{2}$ is the sample size, and the degrees of freedom are equal to $\mathrm{n}_{2}-1$. According to the null hypothesis being tested (i.e., that both the single specimen and the sample come from similar populations), $\mu 1$ and $\mu 2$ (the population means), are also deemed identical. 


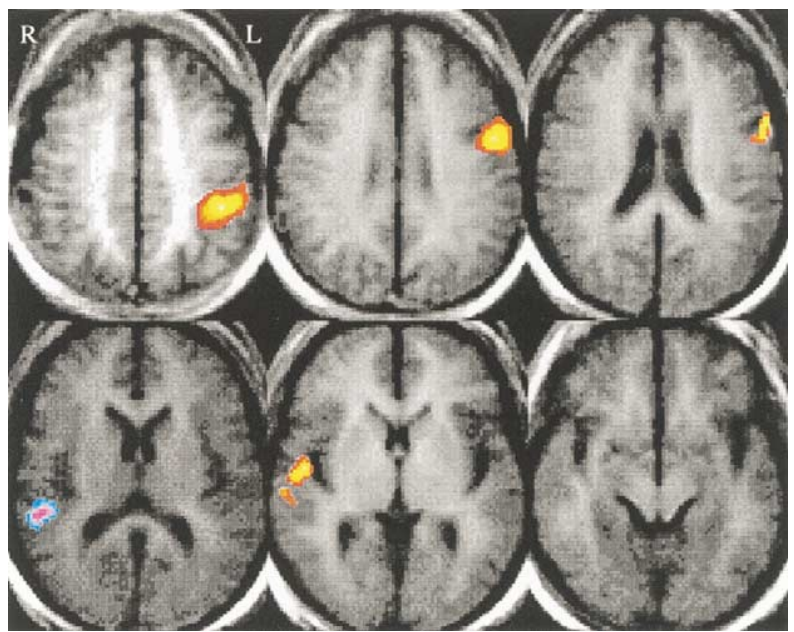

Fig. 3. Average activation of AP control subjects after conversion of data into Talairach space. Images are shown for Talaraich slices 4 (lower right; $\mathrm{z}=-5.4$ ) to 9 (upper left; $\mathrm{z}=41.1$ ). Red/yellow indicates regions of heightened activation during performance of the musical task. Blue/purple indicates regions of heightened activation during performance of the control task. Statistical analysis was performed using ROI analysis.

\section{Results}

\subsection{AP test}

ML correctly responded to 59 of the 60 stimuli on the AP test $(\bar{X}=.983)$, thus corroborating her claim that she possessed AP. Control subjects performed at an average of .90 \pm .07 (range .84-1.00). This difference was not statistically significant (transformed $t$ test [33]; $\mathrm{t}(4)=1.1$, n.s.), suggesting that ML had absolute pitch capabilities that were similar to those of the reference AP subjects.

\subsection{Behavioral data}

ML performed at $.98, .76$, and .50 for the easy, medium, and hard conditions (compared to $.88 \pm .11, .76 \pm .23$, and $.37 \pm .16$ for the other AP musicians). These differences were not statistically significant $(\mathrm{t}(4)=.83 ; \mathrm{t}(4)=.031$; $\mathrm{t}(4)=.76)$, suggesting that ML performed similarly to the seeing AP subjects in the "moveable do" solfeggio task that was performed in the scanner. In the control task, ML performed at .48 (compared to $.43 \pm .04$ for the rest of the AP musicians).This difference was not statistically significant (transformed $t$ test; $\mathrm{t}(4)=1.43$ ).

\section{3. fMRI data}

Fig. 3 is a composite map for the reference group showing the average activations for the musical task (red/yellow) as compared to the average activation for performance of the control task (blue/purple). Prominent foci of activation were found in the right superior temporal gyrus (STG; BA 22), including secondary auditory cortices, the left SMG

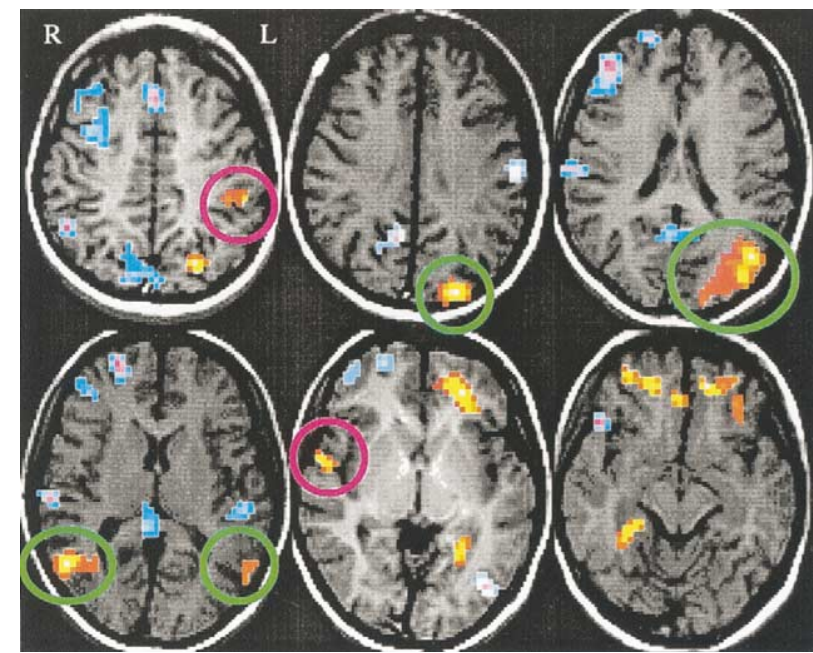

Fig. 4. Comparable slices are shown for subject ML (corresponding to Talaraich z-values of $0.0,9.0,18.1,27.2,36.2$, and 45.3). Red/yellow indicates regions of heightened activation during performance of the musical task and blue/purple indicates regions of heightened activation during performance of the control task. Statistical analysis was performed using ROI analysis. Regions of similar activation between the AP reference subjects and subject ML are circled in pink. Regions of significantly enhanced activation in subject ML are circled in green.

(BA 40), and the left IFG (BA 44/45). Because of the small sample size, statistical significance of subject activations was determined using an ROI analysis, discussed later.

Data for subject ML are shown in Fig. 4, where red/ yellow indicates regions of heightened activation during the musical task, and blue/purple shows regions of heightened activation during performance of the control task. Prominent foci of activation were present in many of the same regions as in the control subjects, including right secondary auditory cortex and the left SMG. Activation in the IFG was not apparent at this threshold. Activations were also found in left extrastriate cortex, the left parietal lobe (angular gyrus), and the orbital-frontal region. As with the control subject images, significance was determined using ROI analysis.

ROI analysis allowed us to compare the extent of functional activation in predefined regions between the sighted AP individuals and for subject ML. ROIs were defined for each of the three areas in the AP network- the right secondary auditory cortex, left IFG, and left SMG- and for the regions that we predicted may be sites of plasticity in blind individuals, including areas of the parietal lobe (the angular gyrus), and the extrastriate cortex (BA 18 and 19). The average cluster size and summed signal intensity (SI) values for each ROI are shown in Table 1. A transformed $t$ test (see above) was used to compare SI values between subject ML and the reference subjects. $\mathrm{t}$-values and p-values are presented in Table 1. For activations that were visible in Figs. 3 and 4, Talaraich coordinates were calculated for the center of activation and those values are included in Table 1, as well.

No differences were found between ML and the refer- 
Table 1

ROI analysis of subject ML and AP controls

\begin{tabular}{|c|c|c|c|c|c|c|c|c|c|}
\hline \multirow[b]{2}{*}{ Region } & \multirow[b]{2}{*}{ Side } & \multicolumn{2}{|c|}{ Talaraich coordinates $\left[\mathrm{t}_{\mathrm{x}}, \mathrm{t}_{\mathrm{y}}, \mathrm{t}_{\mathrm{z}}\right]$} & \multicolumn{2}{|c|}{ Cluster size (voxels) } & \multicolumn{2}{|c|}{ ¿signal intensity (SI) } & \multirow[b]{2}{*}{ t-value } & \multirow[b]{2}{*}{ p-value } \\
\hline & & AP avg. & Subj. ML & AP avg. & Subj. ML & AP avg. & Subj. ML & & \\
\hline $2^{\circ}$ Aud. Cortex & $\mathrm{R}$ & $50.7,-8.9,4.6$ & $54.5,-2.0,4.6$ & $138 \pm 68$ & 144 & $188 \pm 132$ & 163 & -.17 & $\mathrm{p}>.30$ \\
\hline SMG (BA 40) & $\mathrm{L}$ & $-43.6,-34.1,42.3$ & $-43.5,-31.3,41.1$ & $220 \pm 70$ & 372 & $279 \pm 142$ & 337 & .37 & $\mathrm{p}>.30$ \\
\hline BA 39 (incl. Ang. Gyr.) & $\mathrm{R}$ & Below Threshold & $46.9,-59.3,18.1$ & $51 \pm 26$ & 233 & $38 \pm 23$ & 330 & 11.76 & $\mathrm{p}<.001$ \\
\hline BA 39 & $\mathrm{~L}$ & Below Threshold & $-54.2,-59.7,18.1$ & $36 \pm 29$ & 276 & $36 \pm 40$ & 537 & 11.32 & $\mathrm{p}<.001$ \\
\hline
\end{tabular}

ence group in the three regions of the AP network, (all t-values $<.75 ; p>0.30$ ). Significant differences were found in the right and left angular gyrus, and in the left extrastriate cortex (all t-values $>4.00 ; p<0.02$ ). In all of these regions subject ML showed activation that was both more widespread and more intense. No difference was found in the right extrastriate cortex $(\mathrm{t}=.77 ; p>0.30)$.

\section{Discussion}

Subject ML showed similar activation to sighted AP musicians in the network previously identified as underlying absolute pitch. Included in this network were the right secondary auditory cortex, the left IFG, and the left SMG. These data suggest that subject ML's absolute pitch may stem from the same cortical network as sighted AP musicians and is not the byproduct of the recruitment of other visual or associative cortex.

In addition to these regions, ML also showed increased activation in visual and parietal association regions. These areas were not activated in the sighted AP subjects and suggest that ML may recruit them as accessory musical processing regions. The visual activations were in left BA 18 and 19. BA 18 corresponds to V2 and V3 and BA 19 includes V3a, V4, and V5. In sighted individuals, these regions play a critical role in integrating signals from the primary visual cortex and the function of these regions is known to be modified by top-down signals $[18,19]$. A number of previous studies have shown that blind individuals may recruit these regions to process both auditory and somatosensory signals $[1,6,10,16,17,29-31,36]$. To the best of our knowledge, however, this is the first report that they may be recruited for the processing of music.

The parietal activations in subject ML were focused in the right and left angular gyri. In sighted individuals, these areas contain multimodal cells that are responsible for integrating signals from visual, somatosensory and auditory systems $[11,35]$. In the reference AP group, there was minimal activation in these regions. This is not surprising in that the task was exclusively auditory and would therefore be unlikely to activate cells that are typically involved in multisensory integration. However, data have shown that in early blind individuals these regions become dominated by nonvisual systems and do not require visual stimulation to be activated $[7,13,15,17,30]$. This is clearly the case in subject ML.

The recruitment of parietal and extrastriate regions by early blind individuals- as exemplified by subject ML- may be accounted for by early competition models of cortical development $[4,20]$. Various data [21] have shown that in early life, the brain initially has a large number of synapses that undergo a period of intense dying off (at approximately 6-12 years in humans). It is thought that the selection process that determines which synapses are maintained and which ones are eliminated takes place by competitive mechanisms. Thus, in the extrastriate and parietal regions of normal individuals, the strongest synapses in early life are primarily connections from basic visual regions. However, in blind individuals there is no visual input into the occipital and parietal lobules. Subsequently, the connections formed to these regions will be weak. Other cortical inputs, such as auditory or somatosensory, that would usually be eliminated may therefore dominate and remain into adult life. Thus, in early blind individuals, regions that are typically devoted to visual stimulus processing may instead function for other sensory modalities. This type of recruitment may account for reports of superior auditory and somatosensory skills in early blind individuals.

While our experimental paradigm was not sensitive to more general musical aptitude, subject ML is extraordinary in this regard. For example, because she is incapable of reading music, she learns all of her parts "by ear" and quickly memorizes them. She is often able to play back an entire piece after hearing it only once or twice. She is also held in the highest regard by her peers for her mastery of many of the intangible aspects of musical performance. In this regard, subject ML embodies the folkloric notion that because she is blind she has been able to develop superior musical abilities. Our data suggest a neurobiological basis for these reports. It is plausible that her remarkable musical aptitude may derive from the recruitment of visual cortical areas, thus allowing her to devote expanded neural networks to the performance of musical tasks.

Case studies have historically played an important role in psychology. Current theory and research on human memory 
is derived from observations of the densely amnesic patient H.M. Other case-studies have played critical roles in developing theories of visual cognition, emotion, and executive functions. Our data illustrate that new technologies such as fMRI can supplement the behavioral observations that are the mainstay of case reports. fMRI of single subjects provides the opportunity to understand the neural changes that accompany unusual talents or behaviors that tend to be rare or otherwise difficult to study. While it is impossible to extrapolate broad conclusions, such data may be useful to either refute or corroborate extant theories. In the present case, theories of neural plasticity predict specific changes that occur in early blind individuals. Our data are consistent with these theories and we extend previous findings by showing that these regions may be specifically recruited for musical processing in a blind musician with absolute pitch.

\section{Acknowledgments}

The authors would like to thank subject ML for her insights and cooperation. The authors would also like to thank Pasko Rakic, Patricia Goldman-Rakic, Robert Fulbright, Carol Weingarten, and Hoi-Chung Leung for their expert consultation on this project, Cheryl Lacadie and Hedy Sarofin for technical assistance, and Thomas C. Duffy and Thomas Cantey for invaluable musical consultation.

\section{References}

[1] Alho K, Kujala T, Paavilainen P, Summala H, Naatanen R. Auditory processing in visual brain areas of the early blind: evidence from event-related potentials. Electroencephalogr Clin Neurophysiol 1993; 86:418-27.

[2] Bachem A. Absolute pitch. J Acoust Soc Am 1955;27:1180-5.

[3] Benguerel AP, Westdal C. Absolute pitch and the perception of sequential musical intervals. Music Perception 1991;9:105-20.

[4] Bourgeois J.-P, Goldman-Rakic P, Rakic P. Formation, elimination and stabilization of synapses in the primate cerebral cortex. In: Gazzaniga MS, editor. Cognitive Neuroscience. A handbook for the field. Cambridge: MIT Press, 1999. pp. 23-32.

[5] Bourgeois JP, Rakic P. Synaptogenesis in the occipital cortex of macaque monkey devoid of retinal input from early embryonic stages. Eur J Neurosci 1996;8:942-50.

[6] Buchel C, Price C, Frackowiak RS, Friston K. Different activation patterns in the visual cortex of late and congenitally blind subjects. Brain 1998;121:409-19.

[7] Carlson S, Pertovaara A, Tanila H. Late effects of early binocular visual deprivation on the function of Brodmann's area 7 of monkeys (Macaca arctoides). Brain Res 1987;430:101-11.

[8] Cohen JD, MacWhinney B, Flatt M, Provost J. PsyScope: a new graphic interactive environment for designing psychology experiments. Behavioral Research Methods Instruments and Computers 1993;25:257-71.

[9] Cohen LG, Celnik P, Pascual-Leone A, Corwell B, Falz L, Dambrosia J, Honda M, Sadato N, Gerloff C, Catala MD, Hallett M. Functional relevance of cross-modal plasticity in blind humans. Nature 1997; 389:180-3.

[10] De Volder AG, Bol A, Blin J, Robert A, Arno P, Grandin C, Michel $C$, Veraart C. Brain energy metabolism in early blind subjects: neural activity in the visual cortex. Brain Res 1997;750:235-44.
[11] Ettlinger G, Wilson WA. Cross-modal performance: behavioural processes, phylogenetic considerations and neural mechanisms. Behav Brain Res 1990;40:169-92.

[12] Gyllensten L, Malmfors T, Norrlin ML. Growth alteration in the auditory cortex of visually deprived mice. J Comp Neurol 1966;126: 463-9.

[13] Hyvarinen J, Hyvarinen L, Linnankoski I. Modification of parietal association cortex and functional blindness after binocular deprivation in young monkeys. Exp Brain Res 1981;42:1-8.

[14] Korte M, Rauschecker JP. Auditory spatial tuning of cortical neurons is sharpened in cats with early blindness. J Neurophysiol 1993;70: 1717-21.

[15] Kujala T, Alho K, Huotilainen M, Ilmoniemi RJ, Lehtokoski A, Leinonen A, Rinne T, Salonen O, Sinkkonen J, Standertskjold-Nordenstam CG, Naatanen R. Electrophysiological evidence for crossmodal plasticity in humans with early- and late-onset blindness. Psychophysiology 1997;34:213-6.

[16] Kujala T, Huotilainen M, Sinkkonen J, Ahonen AI, Alho K, Hamalainen MS, Ilmoniemi RJ, Kajola M, Knuutila JE, Lavikainen J. Visual cortex activation in blind humans during sound discrimination. Neurosci Lett 1995;183:143-6.

[17] Liotti M, Ryder K, Woldorff MG. Auditory attention in the congenitally blind: where, when and what gets reorganized? Neuroreport 1998;9:1007-12.

[18] Luck SJ, Chelazzi L, Hillyard SA, Desimone R. Neural mechanisms of spatial selective attention in areas V1, V2, and V4 of macaque visual cortex. J Neurophysiol 1997;77:24-42.

[19] Martinez A, Anllo-Vento L, Sereno MI, Frank LR, Buxton RB, Dubowitz DJ, Wong EC, Hinrichs H, Heinze HJ, Hillyard SA. Involvement of striate and extrastriate visual cortical areas in spatial attention. Nat Neurosci 1999;2:364-9.

[20] Rakic P. Specification of cerebral cortical areas. Science 1988;241: $170-6$.

[21] Rakic P, Bourgeois JP, Eckenhoff MF, Zecevic N, Goldman-Rakic PS. Concurrent overproduction of synapses in diverse regions of the primate cerebral cortex. Science 1986;232:232-5.

[22] Rakic P, Lidow MS. Distribution and density of monoamine receptors in the primate visual cortex devoid of retinal input from early embryonic stages. J Neurosci 1995;15:2561-74.

[23] Rakic P, Suner I, Williams RW. A novel cytoarchitectonic area induced experimentally within the primate visual cortex. Proc Natl Acad Sci USA 1991;88:2083-7.

[24] Rauschecker JP, Harris LR. Auditory compensation of the effects of visual deprivation in the cat's superior colliculus. Exp Brain Res 1983;50:69-83.

[25] Rauschecker JP, Korte M. Auditory compensation for early blindness in cat cerebral cortex. J Neurosci 1993;13:4538-48.

[26] Roder B, Rosler F, Neville HJ. Effects of interstimulus interval on auditory event-related potentials in congenitally blind and normally sighted humans. Neurosci Lett 1999;264:53-6.

[27] Ross DA, Stevens AA, Fulbright RK, Gore JC. Neural networks involved in intervalic pitch perception. Breckenridge, Colorado: Neural Integration Processing Systems, 1999.

[28] Ryugo DK, Ryugo R, Globus A, Killackey HP. Increased spine density in auditory cortex following visual or somatic deafferentation. Brain Res 1975;90:143-6.

[29] Sadato N, Hallett M. fMRI occipital activation by tactile stimulation in a blind man. Neurology 1999;52:423.

[30] Sadato N, Pascual-Leone A, Grafman J, Deiber MP, Ibanez V, Hallett M. Neural networks for Braille reading by the blind [see comments]. Brain 1998;121:1213-29.

[31] Sadato N, Pascual-Leone A, Grafman J, Ibanez V, Deiber MP, Dold G, Hallett M. Activation of the primary visual cortex by Braille reading in blind subjects [see comments]. Nature 1996;380:526-8.

[32] Skudlarski P, Constable RT, Gore JC. ROC analysis of statistical methods used in functional MRI: individual subjects. Neuroimage 1999;9:311-29. 
[33] Sokal RR, Rohlf FJ. Biometry. The principles and practice of statistics in biological research. 2nd Ed. WH Freeman and Company, 1981: p 859.

[34] Talairach J, Tournoux P, Missir O. Referentially Oriented Cerebral MRI Anatomy. Thieme Medical Publishers, Inc., 1993: p 234.

[35] Thompson RF, Shaw JA. Behavioral correlates of evoked activity recorded from association areas of the cerebral cortex. J Comp Physiol Psychol 1965;60:329-39.
[36] Uhl F, Franzen P, Podreka I, Steiner M, Deecke L. Increased regional cerebral blood flow in inferior occipital cortex and cerebellum of early blind humans. Neurosci Lett 1993;150:162-4.

[37] Vidyasagar TR. Possible plasticity in the rat superior colliculus. Nature 1978;275:140-1.

[38] Welch GF. Observations on the incidence of absolute pitch (AP) ability in the early blind. Psychology of Music 1988;16:77-80. 